\title{
A remote-control datalogger for large-scale resistivity surveys and robust processing of its signals using a software lock-in approach
}

\author{
Frank Oppermann and Thomas Günther \\ Leibniz Institute for Applied Geophysics, Hannover, 30655, Germany \\ Correspondence: Frank Oppermann (frank.oppermann@liag-hannover.de)
}

Received: 16 June 2017 - Discussion started: 3 July 2017

Revised: 13 December 2017 - Accepted: 19 December 2017 - Published: 8 February 2018

\begin{abstract}
We present a new versatile datalogger that can be used for a wide range of possible applications in geosciences. It is adjustable in signal strength and sampling frequency, battery saving and can remotely be controlled over a Global System for Mobile Communication (GSM) connection so that it saves running costs, particularly in monitoring experiments. The internet connection allows for checking functionality, controlling schedules and optimizing pre-amplification. We mainly use it for large-scale electrical resistivity tomography (ERT), where it independently registers voltage time series on three channels, while a square-wave current is injected. For the analysis of this time series we present a new approach that is based on the lock-in (LI) method, mainly known from electronic circuits. The method searches the working point (phase) using three different functions based on a mask signal, and determines the amplitude using a direct current (DC) correlation function. We use synthetic data with different types of noise to compare the new method with existing approaches, i.e. selective stacking and a modified fast Fourier transformation (FFT)-based approach that assumes a $1 / f$ noise characteristics. All methods give comparable results, but the LI is better than the well-established stacking method. The FFT approach can be even better but only if the noise strictly follows the assumed characteristics. If overshoots are present in the data, which is typical in the field, FFT performs worse even with good data, which is why we conclude that the new LI approach is the most robust solution. This is also proved by a field data set from a long 2-D ERT profile.
\end{abstract}

\section{Introduction}

In geosciences there is a high demand of data from observations of different sorts. Particularly, understanding processes requires monitoring various variables with a high temporal resolution. Typical fields are hydrology, geochemistry and geophysics. Consequently, there is a variety of solutions, mostly for very specific purposes. Dataloggers are often installed in regions without a power supply and must therefore have low battery consumption. Furthermore, communication with dataloggers is beneficial when checking their functionality or even to adjusting settings such as pre-amplification, sampling time or wake-up times.

Autarkic dataloggers are very beneficial in large-scale DC resistivity surveys. ERT (electrical resistivity tomography) is a standard exploration and monitoring technology for environmental and engineering problems owing to its high resolution and low cost. The target parameter electrical resistivity and its inverse electrical conductivity exhibit high sensitivity to important variables such as groundwater salinity or clay content. So-called multi-electrode instruments are popular where a multiple-wired cable connects a number of electrodes. Two electrodes are used to inject a current and two others to measure a voltage. By varying these combinations, hundreds or thousands of measurements are easily conducted per hour. However, the use of these multi-electrode systems is limited to rather small layouts, typically in the order of about 100 electrodes with maximum electrode distances of about $10-20 \mathrm{~m}$. This restricts the depth of penetration to a few hundreds of metres.

In order to achieve deeper signal penetration, one can use dipole-dipole experiments, in which both current injection and voltage measurement are realized by dipoles that are small compared to the total layout (Alfano 1974). Usually, 
square-wave signals are injected, because the signal can easily be recognized. Alfano (1980) imaged geological structures in dipole-dipole experiments. The longest measured profiles measure up to $22 \mathrm{~km}$ length: Storz et al. (2000) imaged fault zones at the German continental deep-drilling site KTB (Kontinentale Tiefbohrung) and Schütze and Flechsig (2002) conducted such a profile across the flanks of the long valley caldera by using a large-scale dipole-dipole experiment to image fluid flow (Pribnow et al., 2003). Friedel (2000) used measurements along the flanks of the Merapi volcano to derive 2-D subsurface images to locate the magma chamber. Günther et al. (2011) described how a fault zone can be imaged with large-scale ERT and structural information from seismics along a $2.5 \mathrm{~km}$ long profile.

However, one can easily extend the technique to three dimensions. Brunner et al. (1999) investigated a Tertiary maar using a layout of each 24 dipoles in three rings. Schünemann et al. (2007) used a star layout to derive apparent resistivity tensors over a buried valley. Similarly, Agricola et al. (2016) used this layout to map the volcanic structure of the Vogelsberg. Flechsig et al. (2010) demonstrated a feasibility dipoledipole test in a $20 \times 20 \mathrm{~km}$ area inside the Eger rift zone, from which a block model could be derived from the sparsely distributed current and voltage dipoles. A surface-downhole ERT survey was used by Bergmann et al. (2012) in the context of carbon dioxide sequestration. Ronczka (2015) used boreholes as long electrodes to investigate inland saltwater intrusion into a $4 \times 4 \mathrm{~km}$ wide area endangered by saltwater upconing (Günther et al., 2015), using a prototype of the datalogger presented here. Before, most large-scale ERT surveys used either high-frequency single-channel seismic dataloggers (e.g. Reftek Texan-125) or three-channel magnetotelluric dataloggers (Roßberg, 2007; Golden et al., 2004). However, the latter provided only a maximum sampling frequency of $8 \mathrm{~Hz}$ leading to anthropogenic noise energy folding into the signal frequency band (typically slightly below $1 \mathrm{~Hz}$ ) due to aliasing effects. As long cables are lying in the field, a significant amount of manpower is required to control whether there are valid recorded voltages or if the cables have been affected by human or animal activity. Furthermore, the preamplification must be set up before the measurements and cannot easily be changed, thus resulting in a loss of either data or accuracy. We address these two shortcomings with a new datalogger that can be controlled by mobile phone communication protocols.

In all these experiments, long time series are recorded and need to be analysed in order to determine the current and voltage strengths. Friedel (2000) compared different approaches and presented a method based on signal stacking using a programme called DC trap that was applied in some cases (e.g. Schütze and Flechsig, 2002; Flechsig et al., 2010; Bergmann et al., 2012). Other approaches (e.g. Günther et al., 2011; Agricola et al., 2017) use the energy of the signals in the Fourier spectrum to retrieve signal strength. Schünemann et al. (2007) used an inverse method to determine the correlation coefficient between the current and voltage. This approach is already related to the lock-in approach, which is also used in some multi-electrode resistivity instruments (e.g. 4point light $10 \mathrm{~W}$ of LGM electronics, www.l-gm.de). Furthermore, an Italian group used deep electrical resistivity tomography (DERT) to successively image several deep geological structures (e.g. Santilano et al., 2015 for an overview). Colella et al. (2004) images the Agri sediment basin in profiles of up to $6.5 \mathrm{~km}$ length. Rizzo et al. (2004) compare these results with small-scale ERT. Tamburriello et al. (2008) revealed geothermally relevant fluid-affected structures. Balacso et al. (2011) give insight into tectonic structures in the area of the disastrous L'Aquila earthquake.

Lock-in detectors working with the principle of phasesensitive detection are widely used to retrieve small signals from a huge noise floor (Meade, 1982, 1983; Blair and Sydenham, 1975). The signal to be measured is modulated by a reference frequency. The receiver "locks in" to this frequency thus reducing the effect of ambient noise (Scofield, 1994). This results in detection at very low signal-to-noise ratios. In the beginning only analogue lock-in amplifiers were implemented with tubes (Baker, 1954, Dereppe, 1961), operational amplifiers (De Marcellis et al., 2012) and applicationspecific integrated circuits (ASIC) (Ferri et al., 2001). Nowadays digital lock-in detectors implemented by discrete circuits (Saam and Conradi, 1998), digital signal processors (DSP) (Sonnaillon and Bonetto, 2005; Proksch, 2006), field programmable gate arrays (FPGA) (Wilson et al., 2015), microcontrollers (Bengtsson, 2012) or software (Andersson et al., 2007) are more common. Digital lock-in detection is more robust compared to analogue solutions. In particular, performances at low frequencies are significantly better. The field of applications which require the detection of very low signals in noisy surroundings where the use of lock-in detectors from optics is widespread (Andersson et al., 2007; Masciotti et al., 2008; Holzman et al., 2005), impedance spectroscopy (Albertini and Kleemann, 1997), wireless networks (Gabal et al., 2010), biologic applications (Ferri et al., 2001; Johnson et al., 2002), electron spin resonance (ESR) (Vistnes et al., 1984; Murányi et al., 2004) to nuclear magnetic resonance (NMR) (Saam and Conradi, 1998; Caracappa and Thorn, 2003).

In the following, we describe layout and usage of the datalogger before presenting a new approach for processing the retrieved time series along with established routines. We use synthetic noisy data to compare the performance of the methods and show the application to field data from a large-scale ERT survey before drawing some conclusions. 


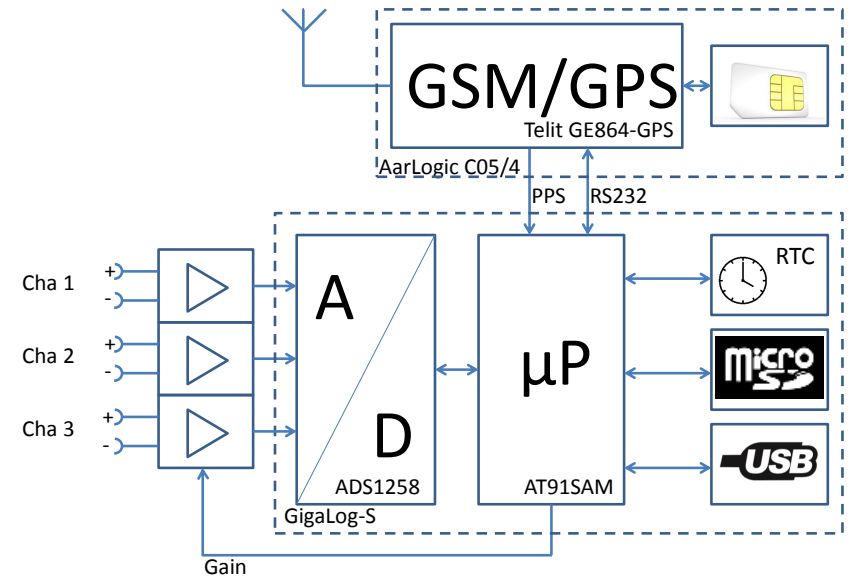

Figure 1. Block diagram of datalogger consisting of a GigaLog-S data acquisition module, a combined GSM/GPS modem and three preamplifiers.

\section{Datalogger}

\subsection{Hardware}

The core of the datalogger (Fig. 1) is a GigaLog-S (Controlord, France) data acquisition module. It contains a 16channel 24 bit delta-sigma A/D converter connected to an Atmel microprocessor. Only three of the channels are used by three DC-coupled high-impedance differential preamplifiers, with four programmable gain stages (factors 2, 10, 20 and 100) that are controlled by the microprocessor. It is vital for good ERT data to optimize the input gain, as ERT signals cover a wide range of magnitudes due to different geometries (source-receiver distance). We designed the datalogger using three channels to record data in all spatial directions generally. For the particular case of 2-D set-ups, where adjacent dipoles in the same direction are measured, it is also a good trade-off between minimizing the length of wires to the electrodes and the number of dataloggers to be installed in the survey area. The sample interval can be between $1 \mathrm{~ms}$ and $1 \mathrm{~s}$ in steps of $1 \mathrm{~ms}$ and is therefore significantly higher than existing MT (Roßberg, 2007) or ERT (Balasco et al., 2008) dataloggers. The sampled data will be stored in a text file on a micro SD card. The memory consumption is about $100 \mathrm{MB} \mathrm{h}^{-1}$ at $1 \mathrm{~ms}$ sample interval. The datalogger can be accessed through a local USB cable or the combined GSM/GPS module AarLogic C05/4 remote via a General Packet Radio Service (GPRS) connection. The core of this module is a Telit GE864-GPS chip. We chose just a GPRS modem to change the parameters and record small pieces of a time series remotely, because our main aim was to minimize power consumption, which is significantly lower than a $3 \mathrm{G}$ or 4G module. The Global Positioning System (GPS) receiver of this module allows the microprocessor clock to be synchronized by setting the time of the realtime clock and saving

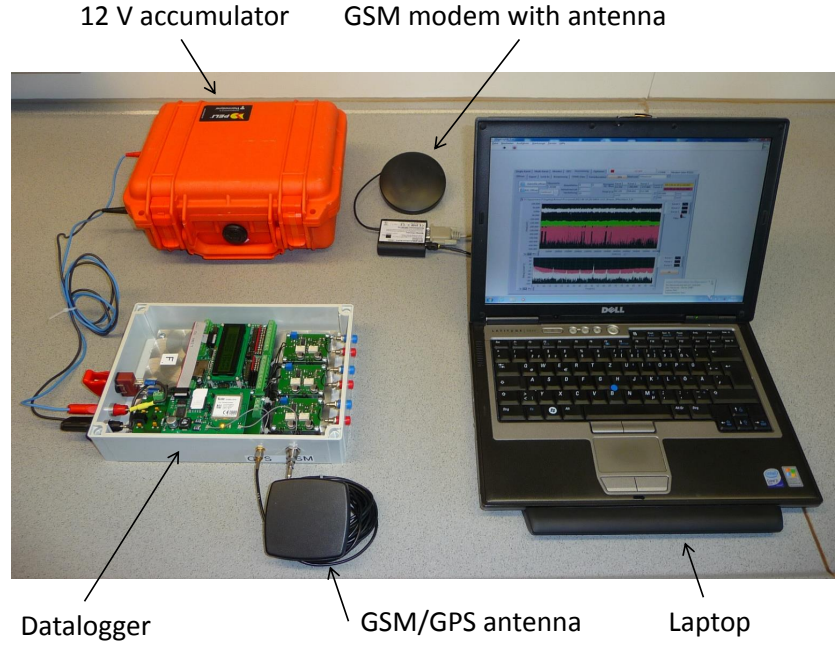

Figure 2. Datalogger system with datalogger, GSM/GPS antenna and $12 \mathrm{~V}$ accumulator. A laptop with GSM modem and antenna for remote control.

the GPS position of the datalogger on the micro SD card. The GPS timing can be transferred by an NMEA format string, which results in synchronization of $1 \mathrm{~s}$ or by the PPS (pulse per second) signal that synchronizes in $1 \mathrm{~ms}$, The system is powered by an external $12 \mathrm{~V}$ accumulator. The power consumption is about $300 \mathrm{~mA}$ with an active GPRS connection, $150 \mathrm{~mA}$ with an idle GPRS connection and $15 \mathrm{~mA}$ in sleep mode. The programmable sleep mode puts the logger system into standby after operating hours. At a specified time, it powers up again, restoring all parameters and synchronizing the GPS time again. Figure 2 shows the system with the datalogger connected to the combined GSM/GPS antenna, a $12 \mathrm{~V}$ accumulator and a laptop with GSM modem and antenna to remotely control the datalogger.

\subsection{Graphical user interface (GUI)}

The software GUI is written in LabView. In the singlechannel mode it is possible to connect to one logger and control the different functions, such as starting or stopping the data acquisition or setting the filename, gain or sampling interval. It is possible to read the directory of the SD card, monitor the accumulator voltage, the temperature of the logger or the free memory of the SD card. Entire data files can be downloaded, but this is only advisable with a USB connection due to the slow transfer rate of the GPRS connection. Another major feature of the remote controllability is that the signals of the acquired data can be monitored in realtime. To control many loggers simultaneously we provide a tabular control (Fig. 3). For a survey setting you can choose the loggers by filename, gain and sample interval in the first four columns. By executing this sequence the online status of every logger will be checked and the parameters will be set and read back. It also checks whether the data acquisition 


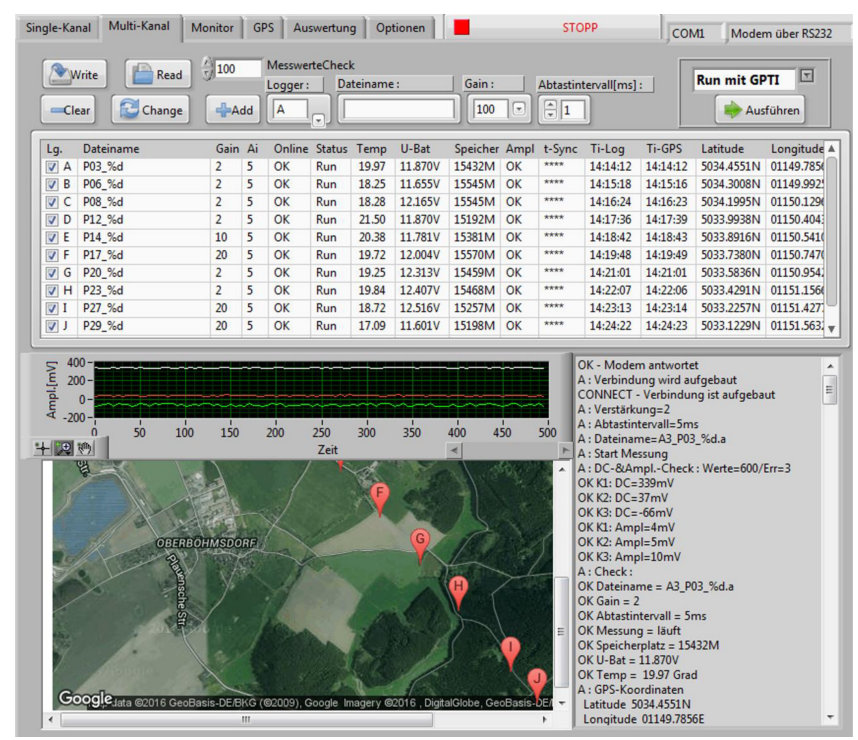

Figure 3. Software GUI in multi-channel mode.

is running and if the logger temperature, accumulator voltage and SD memory are within predefined values. A small amount of data are downloaded and it is checked that the input signals of the three channels are in the proper range of amplitude and offset voltage. The datalogger time is checked for recent synchronization with GPS time. GPS coordinates are displayed and a Google map image is created to show the datalogger positions. It is also important to set the shut-down and wake-up times for the sleep mode.

Figure 4 shows a sample signal containing the response of an injected $0.2 \mathrm{~Hz}$ ERT square-wave current as visible in the GUI monitoring software. A fast Fourier transform (FFT) algorithm calculates the corresponding frequency spectrum. On top of the underlying broadband noise one can see the $50 \mathrm{~Hz}$ signal of the power lines, the $16.7 \mathrm{~Hz}$ from the railway lines, and the $0.2 \mathrm{~Hz}$ ERT signal with their odd harmonics. Colella at al. (2004) also shows a frequency spectrum of a time series.

\section{Signal processing of ERT signals}

The most common approach to retrieve the amplitude of an ERT square-wave signal from the recorded time series is a stacking algorithm, as used by some multi-electrode instruments (e.g. RESECS by http://www.geoserve.de/). Other instruments (e.g. 4point light $10 \mathrm{~W}$ by http://www.l-gm.de) use (hardware) lock-in amplifiers, which show very good results at very low signal-to-noise ratios. Thirdly, signals can be analysed in the Fourier domain by relating the FFT energy of both current and voltage spectrum (e.g. Agricola et al., 2017). We process the recorded time series using all three approaches, including a newly developed lock-in method. To validate the quality of the latter approach, we compare its re- sults with stacking and FFT algorithms. As there are changes in the implementation, we describe and illustrate all three variants in the following. We assume a square-wave signal of $0.2 \mathrm{~Hz}$ for synthetic studies. This is also used in the field as a trade-off between stability and noise impact: a half period of $2.5 \mathrm{~s}$ provides enough samples for a robust amplitude determination. Higher frequencies would reduce the amount of samples so that the unstable part of the transient effects can dominate. Smaller frequencies would probably lead to smaller signal-to-noise ratios due to the typical $1 / f$ noise characteristics.

\subsection{FFT}

For the processing we use the frequency spectrum of the recorded time series. This power spectrum is created by a fast Fourier transformation (FFT) using a Gaussian window. If the duty cycle of the square-wave signal is $50 \%$, the frequency power spectrum is

$$
P(t)=\frac{4}{\pi} \sum_{n=1}^{\infty}\left(\frac{\sin (2 \pi(2 n-1) f t)}{2 n-1}\right) .
$$

We focus on the first harmonic of the ERT signal. However, we cannot just take the value at the first harmonic as broadband noise also contains significant values at this frequency. To determine the effect of this noise, we have to subtract the noise level around the first harmonic (Fig. 5). To calculate the broadband noise level we cut out all harmonics of the ERT signal and consider only the frequency range from DC to the 15th harmonics. Unlike Günther et al. (2011) or Agricola et al. (2017) we do not assume a constant noise floor over the frequency axis but assume a $1 / f$ decrease that is often observed (Surkov and Hayakawa, 2007). Therefore we carry out a non-linear fit with a noise model consisting of a noise floor and an inverse $f$ contribution:

$u(f)=a 0+\frac{a 1}{a 2+f}$

The peak amplitude Up of the ERT square-wave signal is then calculated by the following Eq. (3) from the peak value $P_{o}$ and the noise value $P_{\text {noise }}$ at the first harmonic.

$\mathrm{Up}=\sqrt{\frac{4}{\pi}\left(P_{o}-P_{\text {noise }}\right)}$.

The quality criterion is the signal-to-noise ratio at the base frequency.

\subsection{Stacking}

There are many different ways to stack a signal (Naess and Bruland, 1985). To keep this comparison simple, we used only one stacking method, the alpha-trimmed-mean stack (Friedel, 2000). The first step is a drift correction (Friedel, 2000). The drift-corrected function $U_{\mathrm{dr}}(t)$ is the time series 

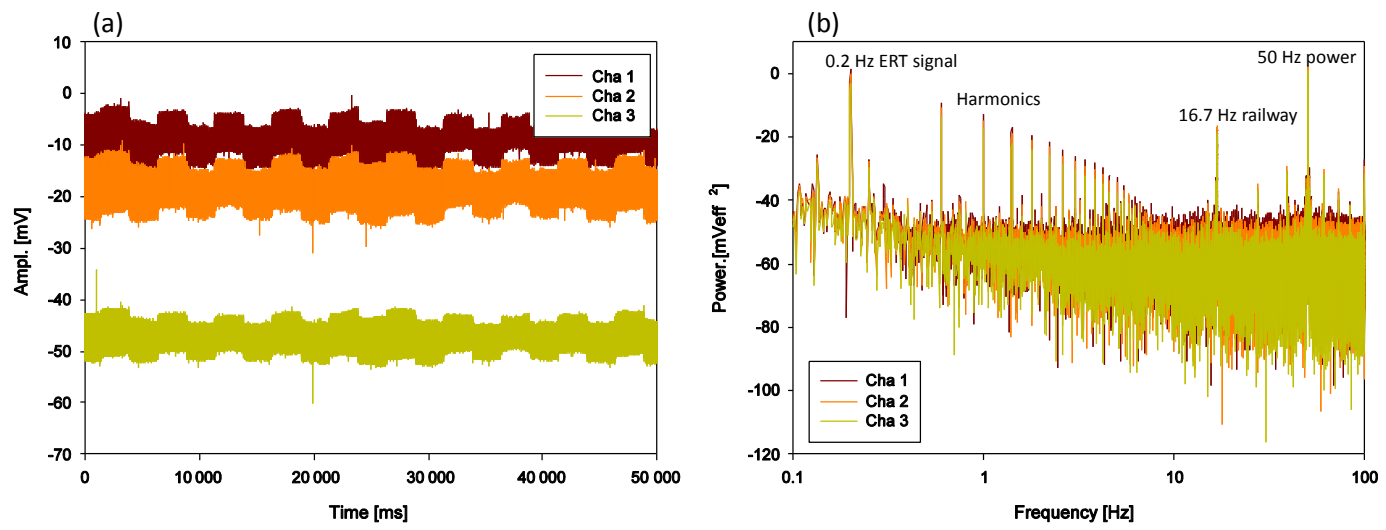

Figure 4. Exemplary voltage time series (a) from a square-wave current injection and frequency spectrum (b) as visible in the GUI monitoring software.

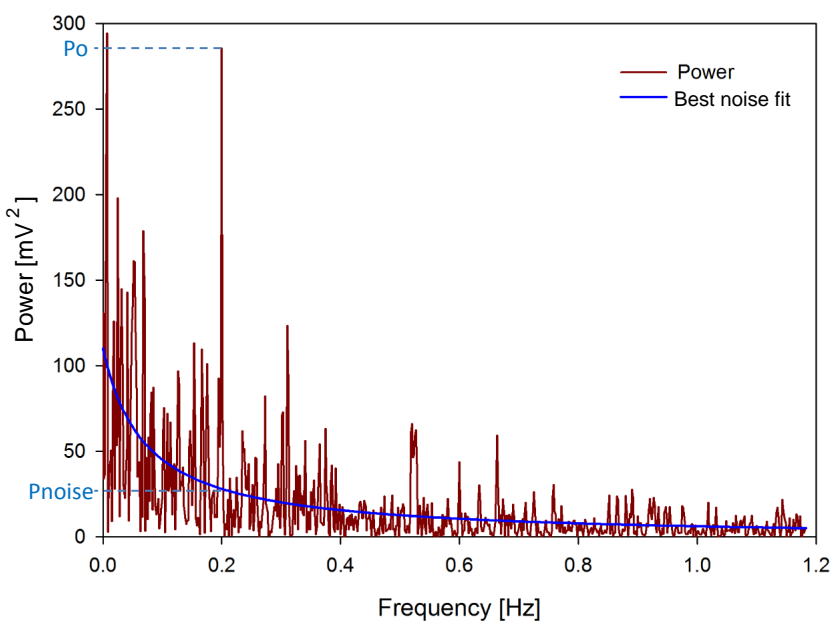

Figure 5. FFT-based fitting of a noise function $\left(P_{\text {noise }}\right)$ and calculation of the signal strength $\left(P=P_{o}-P_{\text {noise }}\right)$ at the first harmonic $f=0.2 \mathrm{~Hz}$.

$U(t)$ subtracted by the moving mean value of the time series with a window size of the period $\mathrm{M}$ of the ERT signal.

$U_{\mathrm{dr}}(t)=U(t)-\frac{1}{M} \sum_{d=-M / 2}^{M / 2} U(t+d)$

This gives only a correct result if the signal is symmetric, which is mostly the case. At every stack point represented by one sample within the signal period, the median values of all stacks are sorted by amplitude. To eliminate outliers, a variable percentage of the largest and smallest amplitudes is rejected (Fig. 6). A rejection (alpha-trim) rate of $10 \%$ shifts the distribution into a linear behaviour so that the mean is less sensitive to outliers. As we start the stacking at a random point, we do not know the correct phase relation yet. A maximum cross-correlation between the stacked signal and an ideal signature will usually point to the leading and tail-

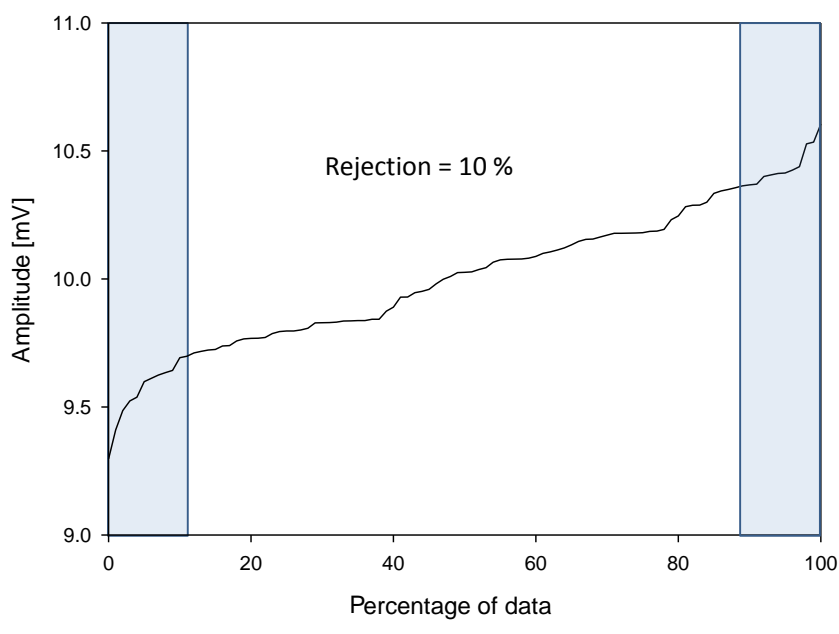

Figure 6. Sorted amplitude distribution for the alpha-trimmedmean stacking.

ing edges. We increase the sensitivity by cross-correlating for all possible phase relations and pick the phase at the highest cross-correlation coefficient. To remove effects like overshoots at the slope of the ERT signal, the average value will be calculated in a variable window of the signal plateau (Fig. 7). The main quality criterion is the ratio of the average values $U_{\text {pos }}$ at the positive and $U_{\text {neg }}$ at the negative plateau. Secondary quality criteria is the maximum cross-correlation coefficient and the mean square error (MSE) of the signal at the plateau compared to the average value.

\subsection{Lock-in}

In the lock-in processing, also known as phase-sensitive rectifying, the negative part of the acquired square wave is rectified, accomplished by a reference signal at the slopes of the square wave. Harmonic noise annihilates itself and disharmonic noise is thus reduced. The DC value of this convo- 


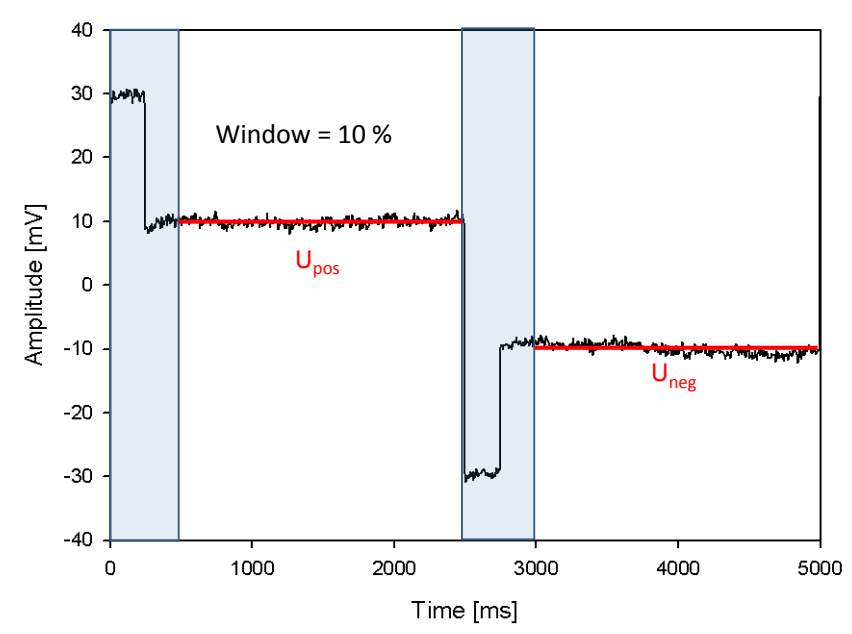

Figure 7. Determination of effective stacking window for calculating the mean value at the plateaus for a synthetic signal containing a $250 \mathrm{~ms}$ overshoot.

luted signal is the desired amplitude. Usually lock-in amplifiers retrieve the hard-wired reference signal from the transmitter in order to have the correct phase information at the receiver. In a survey area of a few square kilometres it is not practical to connect all dataloggers with trigger cables to the current source and so destroy the advantage of the autonomous remote-controlled datalogger. However, we show that it is also possible to solve this problem numerically.

First step is a drift correction (see Sect. 3.2). With a sample interval of $N$ milliseconds and a period of $M$ milliseconds of the injected current we have a number of $P=M \times N^{-1}$ possible phase relations between the acquired and the reference signal. If we generate an artificial numeric reference signal $M(t)$ and convolute this with the time series (Fig. 8) of the received drift-corrected signal $U_{\mathrm{dr}}(t)$ for every phase relation $i=0$ to $P$, we get the convoluted signal $U_{\mathrm{c}}$ Eq. (5).

$U_{\mathrm{c}}(t, i)=U_{\mathrm{dr}}(t) \cdot M(t+i)$ if $M(t+i)<>0$

To remove the effects of overshoots at the slopes, the reference signal $M(t)$ has, additionally to the usual states +1 and -1 , a third state 0 . When $M(t)=0$ the values of the received signal $U_{\mathrm{dr}}(t)$ are not included in the convolution. By calculating the DC value $\mathrm{DC}(i)$ (Eq. 6), the peak-to-peak voltage $\operatorname{Vpp}(i)$ (Eq. 7) and the effective value $\operatorname{rms}(i)$ (Eq. 8) of the convolution $U_{\mathrm{c}}(i)$ we obtain three functions (Fig. 9) from which it is possible to derive the correct phase relation.

$$
\begin{aligned}
& \operatorname{DC}(i)=\frac{1}{N} \sum_{t=0}^{N} U_{\mathrm{c}}(t, i) \\
& \operatorname{Vpp}(i)=U_{\mathrm{c}}(t, i)-U_{\mathrm{c}}(t, i) \\
& \operatorname{rms}(i)=\sqrt{\frac{1}{N} \sum_{t=0}^{N} U_{\mathrm{c}}(t, i)^{2}}
\end{aligned}
$$

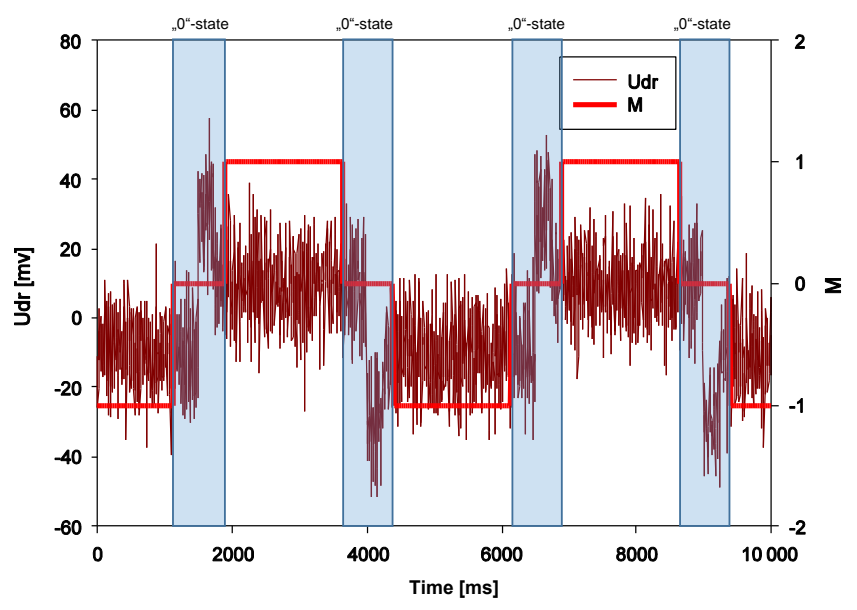

Figure 8. Drift-corrected signal $U_{\mathrm{dr}}(t)$ and mask signal $M(t)$ in correct phase.

The main criteria used to obtain the real phase is the maximum of the DC function within a specific DC search area (THdc threshold of DC function, usually $25 \%$ ). At an ideal square-wave signal the DC value of the convoluted signal is the desired amplitude. As this can lead to the wrong results for signal contributions like overshoots, we additionally see whether there is also a minimum of the Vpp function within a Vpp search area (THvpp threshold of Vpp function, usually $20 \%$ ). The LabView function for the Vpp amplitude calculates the positive and negative peak values from a histogram statistics that can cause the minimum of the Vpp function to be wide. To find the real phase within a wide Vpp minimum, the minimum of the rms function below the rms search area (THrms threshold of rms function, usually $10 \%$ ) is used because the rms value is very sensitive. This procedure is shown in the flow diagram in Fig. 10a and on the example in Fig. 10b: the minimum of the quadratic sum eval $(i)$ of the three normalized criteria functions determines the phase, whereas we find the amplitude in the DC function DC $(i)$. The convolution of the acquired square-wave signal with the reference square-wave signal (mask signal in Fig. 8) results in a triangle-shaped function $\mathrm{DC}(i)$. The linearity of the triangle slope is correlated to the quality of the acquired squarewave signal shape. The mean square error MSE between the triangle signal and a fitted straight line is a high-quality criterion for the lock-in result. We used only the area between 20 and $80 \%$ for the fit to avoid errors caused by overshoots. The MSE can also be used to find the perfect value of the 0 state. To find the best cutting point of transient effects and to determine the plateau of the signal it is advisable to convolute it with different percentages of the $M(t)=0$ state and see where the MSE has a minimum (Fig. 11). At a 0 state of about $25 \%$ the MSE has its minimum. The signal is stable at $10 \mathrm{mVp}$ at the plateau from 0 state 16 to $25 \%$. Below $16 \%$ it does not cut the overshoot appropriately. Too high 


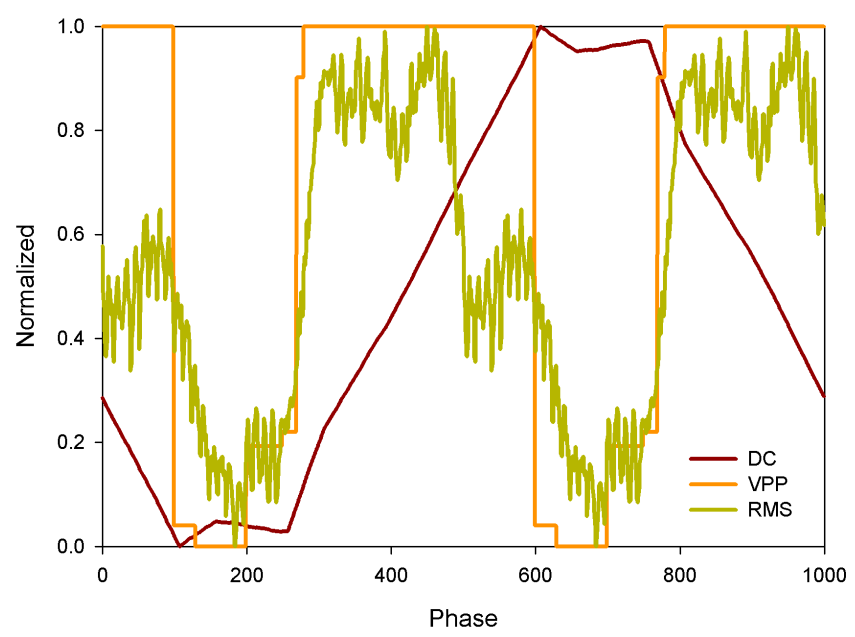

Figure 9. Evaluated functions of direct current (DC) value, peakto-peak voltage Vpp and effective root mean square (rms) of the mask-convoluted signal.

percentages of the 0 state lower the sensitivity of the lock-in detection because the noise reduction effect of the method decreases.

\section{Quality assessment of the processing methods}

\subsection{Synthetic data}

To compare the results of the lock-in-, stacking- and FFTprocessing we created two artificial data sets. Both data sets exhibit a square-wave signal with a frequency of $0.2 \mathrm{~Hz}$ using a $50 \%$ duty cycle and an amplitude of $10 \mathrm{mVp}$. The first data set has a $10 \mathrm{mV}$ overshoot (5\% duty cycle wide) at the leading and trailing edges of the added signal. The second data set is without overshoot. To create realistic noise conditions, we added $75 \mathrm{mV}$ of railway noise at $16.7 \mathrm{~Hz}$ and $100 \mathrm{mV}$ of power noise at $50 \mathrm{~Hz}$. The variable noise source was a pink noise with increasing effective noise amplitude from 0 to $249 \mathrm{mVrms}$ added to both signals. The pink noise is created from white noise filtered through a $1 / f$ digital filter with 0.1 and $100 \mathrm{~Hz}$ lower and upper cut-off frequencies. Each value is the average of 200 processing results with different seeds for the random generator.

\subsection{Comparison of methods}

Each processing has different kind of quality criteria to judge whether a result is valid or not. At the lock-in it is the mean square error of the slope of the DC function. At the stacking it is the ratio of the positive and the negative plateau amplitudes and at the FFT it is the spectral signal-to-noise ratio at the base frequency. For the comparison we adjusted the threshold values of the different quality criteria so that an equal number $(30 \%)$ of invalid results were rejected. This leads of course to an overestimation towards higher noise values of all three methods, because at low amplitudes the tendency to be rejected by the quality criteria is higher. Figure 12 shows the three different quality criteria over noise amplitude in a normalized graph. The criteria for FFT, lock-in and stacking show an exponential increase. Figure 13 shows the results of the comparison of the three processing algorithms for the two data sets without (a) and with (b) overshoot. For lownoise amplitudes all methods yield similar results except the FFT at the data set with overshoots, because at the frequency spectrum we cannot separate between the fractions from the ERT signal and the overshoot. Lock-in and stacking cut off overshoots at the signal slopes and are therefore not sensitive to transient effects if the 0 state has been chosen appropriately (typically $20 \%$ ). At higher noise amplitudes stacking overestimates the signal more than lock-in and FFT. Stacking bases the amplitude decision on a wider-frequency spectrum, while lock-in and FFT just look at a narrow-frequency band. Lock-in and FFT are therefore less affected by broadband noise. Note that the performance of FFT might be overestimated as we applied a noise model that perfectly matches the $1 / f$ behaviour in the FFT approach.

\subsection{Relation of quality factor to $S / N$}

The quality criteria MSE of lock-in data of the synthetic data over noise can be used to validate the quality of the processed data. If we assume the function of MSE over noise (Fig. 12) valid for realistic signal amplitudes we can normalize the signal-to-noise ratio with the signal amplitude of $10 \mathrm{mV}$ :

$\mathrm{MSE}=f($ noise $)=f(S / N)$.

Then we fit this function Eq. (9) with an exponential fit, because this function describes the relation sufficiently well in the relevant $S / N$ range:

$\mathrm{MSE}=a \cdot e^{-(b \cdot S / N[\mathrm{~dB}])}$.

Now it is possible to calculate the signal-to-noise ratios of data from the matching MSE values with the fitting results $a=0.0271$ and $b=0.2949$. This means that for a zero signal $(S / N$ of 1 equaling $0 \mathrm{~dB})$ we obtain an MSE of 0.027 and an increase to about 10 for a $S / N$ of about $-20 \mathrm{~dB}$ ( $S / N$ of 0.1$)$. For any MSE we can thus reconstruct a $S / N$ by rearranging Eq. (10):

$S / N[\mathrm{~dB}]=-\frac{1}{b} \cdot \ln \left(\frac{\mathrm{MSE}}{a}\right)$.

\section{Field case application}

We apply the developed datalogger and analysis methods to a large-scale ERT field case measured near the town of Schleiz in Thuringia, Germany. We focus on the second part of the field campaign and on the part of the profile and remove data 
(a)

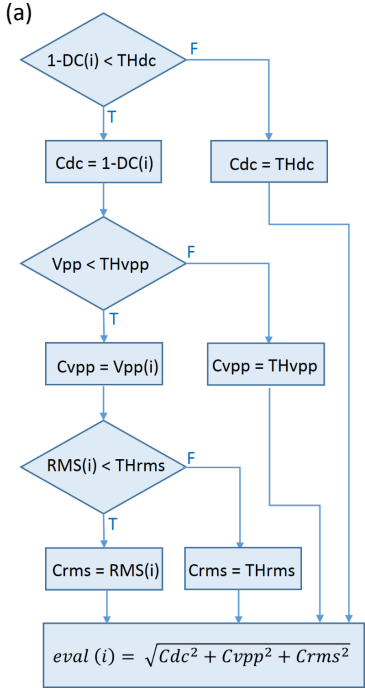

(b)

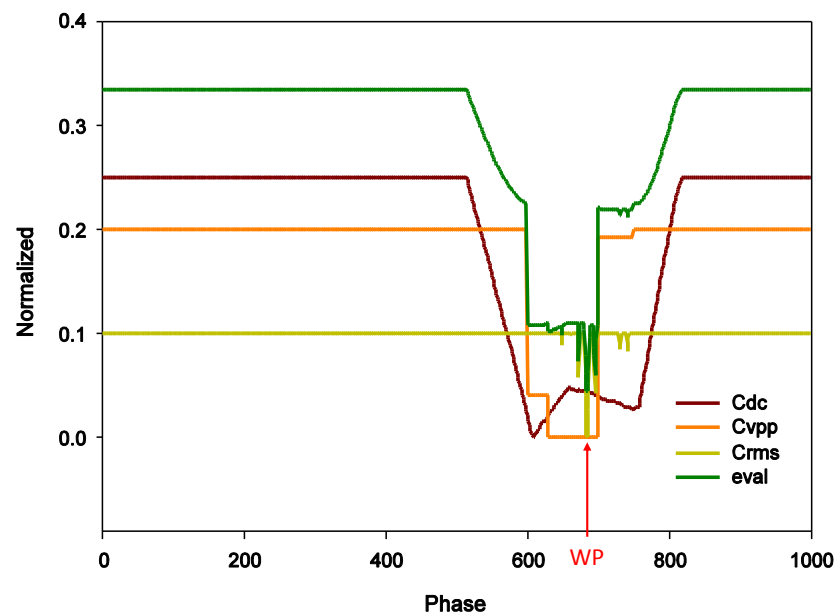

Figure 10. (a) Flow diagram calculation of the components Cdc, Cvpp and Crms from the three functions. (b) Components and quadratic sum for calculating the working point (WP) from its minimum.

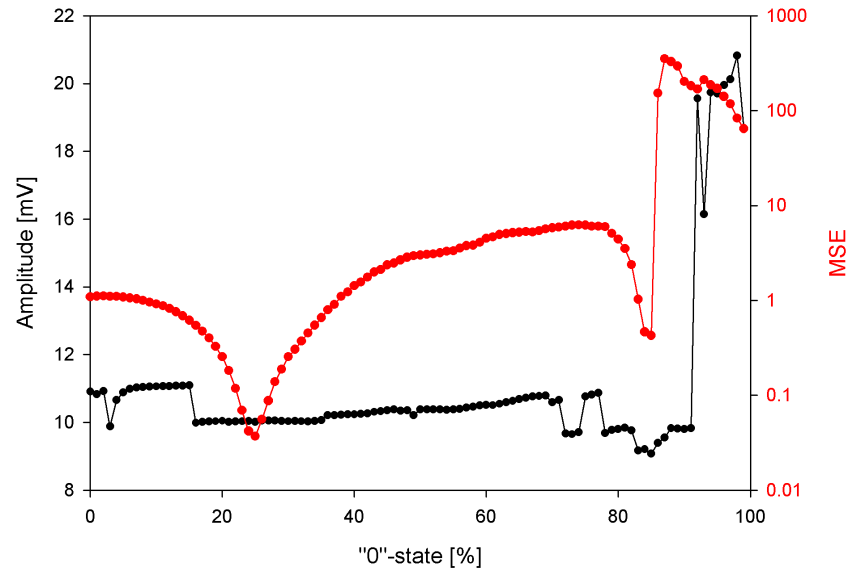

Figure 11. Detected amplitude and mean square error MSE of an artificial signal with $10 \mathrm{mV}$ overshoot and $29 \mathrm{mVrms}$ pink noise over length of 0 state, showing the plateau between 16 and $35 \%$. Up to $16 \% 0$ state is the effect of the overshoot; above $70 \%$ is the reduced noise rejection.

from two other dataloggers. The slightly reduced profile thus consists of 31 electrodes with spacings of $125 \mathrm{~m}$. We used a high-current generator to maximize the magnitude of the measured voltages. Into each dipole we injected the maximum current, ranging from 3 to $25 \mathrm{~A}$, depending on coupling conditions. As for close dipoles, the measured voltage exceeded the input range, we additionally injected a small current of about 2-3 A. Here we only used these data for reasons of homogeneity and the greater $S / N$ range.

A complete dipole-dipole survey was carried out. The principle of reciprocity states that interchanges current and dipole does not change the measured impedance (Friedel,

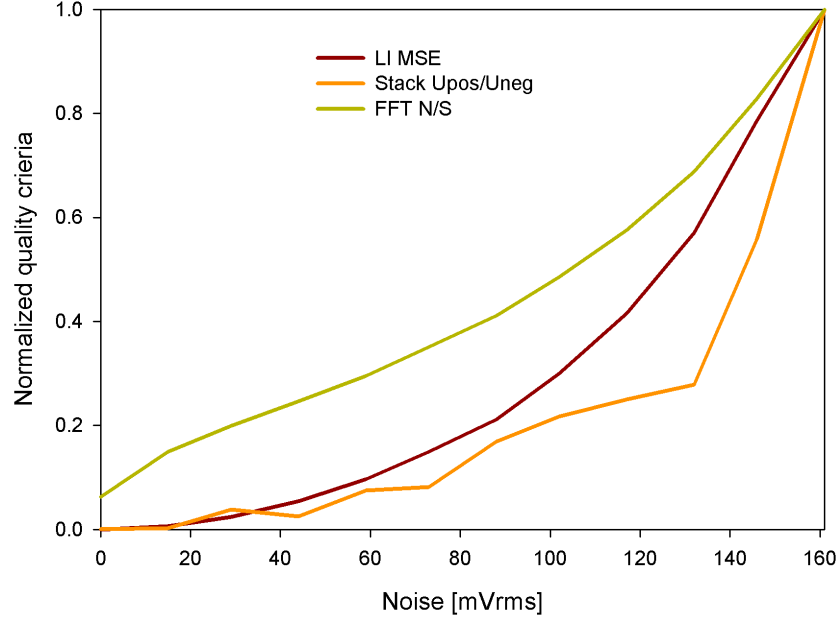

Figure 12. Quality criteria over noise level of test data set without overshoot.

2000). As a consequence, reciprocal measurements can be used for error estimation. For single data this is a checks how the two independent measurements (a forward and a backward dipole-dipole) are combined before going into the inversion routine. For multi-electrode data it has become common practice to use reciprocal data in a statistical sense to derive error models for weighting data in inversion routines (Udphuay et al., 2011). An error model consists of a constant relative error and a voltage error to take the magnitude of the data into account. It is achieved by distributing the data into bins of similar voltage and fitting a curve to the standard deviation of the reciprocal error. As another quality check we injected two different current strengths. The proportion of the 
(a)

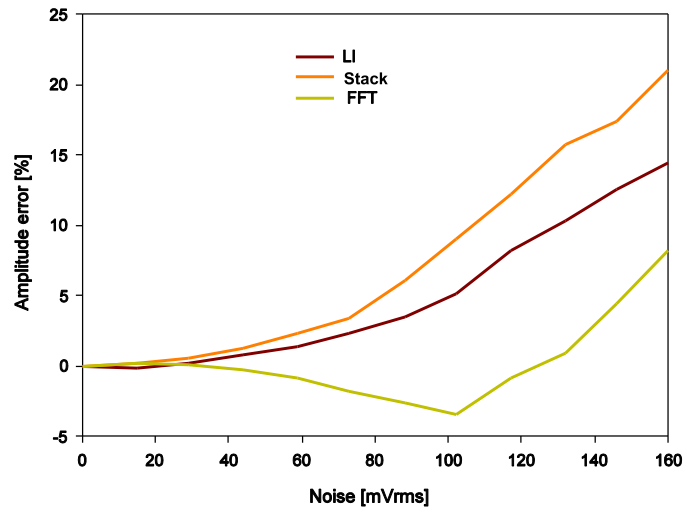

(b)

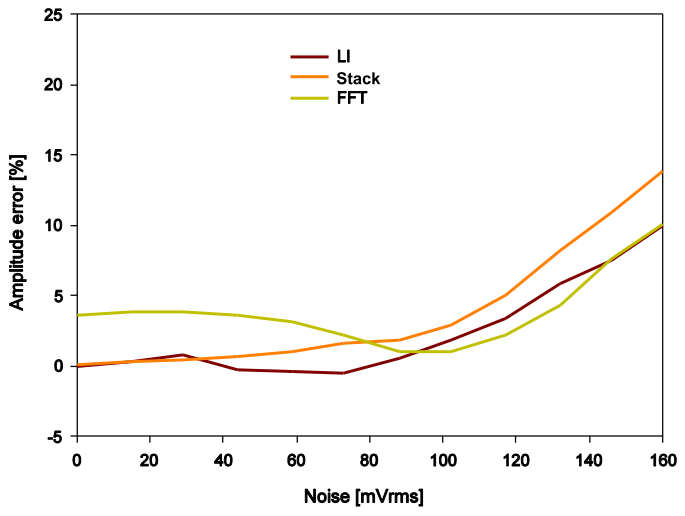

Figure 13. (a) Comparison of LI, stack and FFT over pink noise without overshoot. (b) Comparison of LI, stack and FFT over pink noise with overshoot.

recorded dipole voltages should be identical to the proportion of the injected currents. The small current does not saturate dataloggers in the vicinity of the current source, and the high current provides enough amplitude for the biggest sourcereceiver distances.

To estimate the error level we use Eq. (11) to calculate the $S / N$ of every data point using the matching quality factor MSE. If we assume, for example, that an amplitude error of $>5 \%$ is not acceptable, we see in Fig. 13a that the errors start growing above $5 \%$ at a noise level of $100 \mathrm{mVrms}$. This results in a signal amplitude of $10 \mathrm{mV}$ to a $S / N$ of $-20 \mathrm{~dB}$. Figure 14a shows the distribution of the signal-to-noise ratio in comparison with the MSE of the LI processing of the synthetic data. The majority of the field data are in the region where the MSE and therefore the amplitude errors are negligible. Just some data below the critical $S / N$ of $-20 \mathrm{~dB}$ have an MSE $>10$ corresponding to amplitude errors $>5 \%$. Figure 14b shows the obtained $S / N$ for forward-directed standard dipole-dipole data in a so-called pseudo-section, i.e. as a function of the array midpoint and the dipole separation factor DD (dipole distance by dipole length) that indicates penetration depth. Generally, the $S / N$ decreases with the dipole separation from about 0 to $20 \mathrm{~dB}$ for the shallowest penetrating data down to -10 to $-30 \mathrm{~dB}$ for the deepest data. A comparison with the very heterogeneous apparent resistivity (Fig. 15) shows that $S / N$ is strongly correlated with the measured voltages which are low above conducting zones.

All three processing approaches were applied to the time series. The resulting voltages were divided by the driving current and multiplied by the analytically known geometric factor resulting in the apparent resistivity which represents the resistivity of an equivalent homogeneous half-space. Additionally, we computed the normal reciprocity from each available pair of forward and backward measured array, i.e. the percentage difference divided by the mean value. Figure 15 shows the pseudo-sections of the apparent resistivity (mean values of forward and backward measured) and reciprocity for the three approaches. The upper images are hardly distinguishable - only a few points show distinct deviations due to the wide range of values between 1 and $1000 \Omega$ M. Only some single points are significantly different from their neighbours. There are only a few values missing for the FFT approach in the area of lowest values (and $S / N$ ). This proves that all methods are generally applicable. The lower row shows the detected reciprocity. There is some systematics that might result from a non-match of current electrodes (large coupling fields) and potential electrodes (small $\mathrm{Ag} / \mathrm{AgCl}$ pots). Very few values are below (light grey) or above (dark grey) the given colour limits. The FFT methods have the largest gaps (least number of reciprocal pairs) and overall the largest absolute values, whereas the lock-in image shows the smoothest image (similar to stack). There are strong similarities between the reciprocity and the derived $S / N$ pseudo-section (Fig. 14b), indicating that the MSE error is able to deliver uncertainty information.

\section{Conclusions}

Remote dataloggers can provide valuable information on the subsurface in multi-source or monitoring experiments. The presented datalogger, powered by ordinary batteries, can register long time series with sampling rates of up to $1 \mathrm{kHz}$. It can be accessed and controlled remotely using GSM connection and can therefore save time for a lot of different geoscientific experiments, e.g. in the fields of environmental and groundwater monitoring, or in applied geophysics.

One example is large-scale ERT surveys for geological investigations that cannot be measured with conventional instruments. The registered voltage time series have to be processed to obtain single voltages and their measuring uncertainties. For the ERT experiments that typically use square waves with periods below $1 \mathrm{~s}$ and $50 \%$ duty cycle, there have been two approaches for data analysis, i.e. stacking and FFT 
(a)

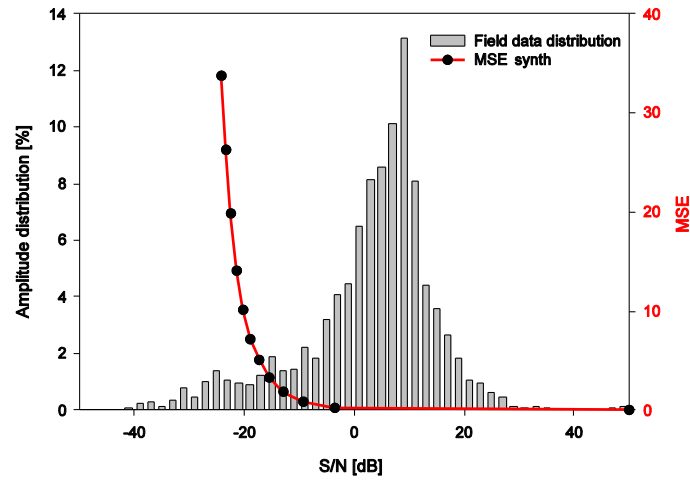

(b)

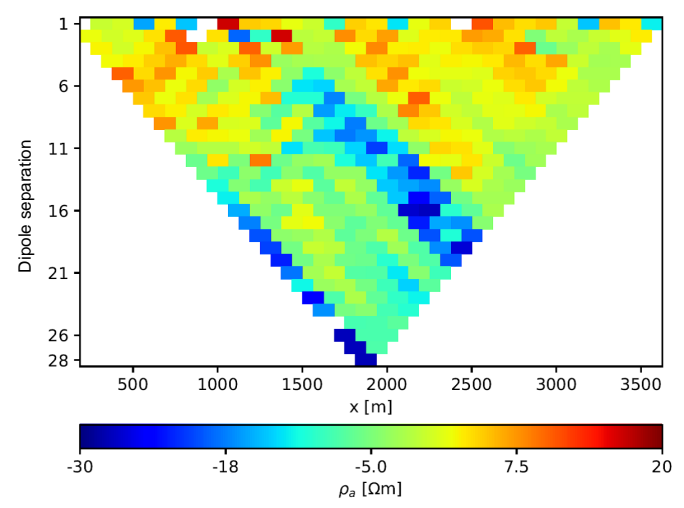

Figure 14. Signal-to-noise values of field data. (a) Histogram compared to MSE of synthetic data, (b) in a pseudo-section.
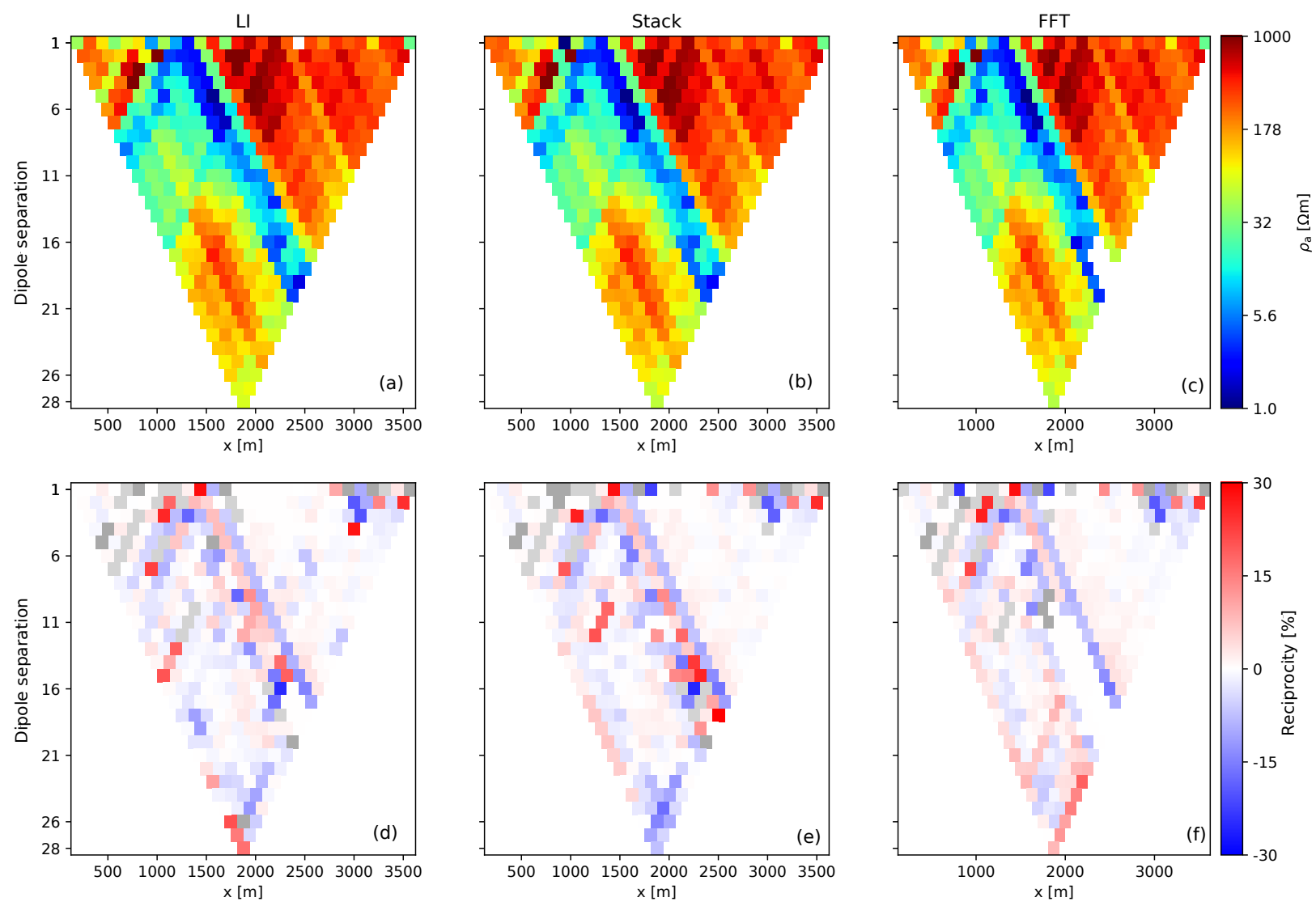

Figure 15. Calculated apparent resistivity $(\mathbf{a}, \mathbf{b}, \mathbf{c})$ and normal reciprocity $(\mathbf{d}, \mathbf{e}, \mathbf{f})$ for the three analysis approaches, lock-in $(\mathbf{a}, \mathbf{d})$, stacking $(\mathbf{b}, \mathbf{e})$ and FFT $(\mathbf{c}, \mathbf{f})$.

methods. In addition, we present a lock-in-based approach, a technique that had been used as hardware solution but can also be applied numerically. To make it robust against different kinds of transient effects and noise, several functions are computed from the convolution with a mask signal. The combination of them is used to determine the working point (phase lag), the voltage amplitude and a measure of uncer- tainty based on a mean square error. The software lock-in processing represents a robust method for determining signal the strength using a reference signal and is thus well suited for ERT.

Synthetic data are used to compare the new method (LI) with an alpha-trimmed stacking and an improved FFT-based method that includes a $1 / f$ noise model. All methods are 
able to retrieve correct voltages down to $S / N$ ratios of about $1 / 10$. In all cases, LI performs slightly better than stacking. The new FFT method can sometimes perform even better than both LI and stacking, but this is due to the fact that the synthetic noise was perfectly $1 / f$, which is not true in reality. Moreover, the often occurring overshoots affect the FFT method even at very good $S / N$, which is why we consider LI to be the most robust method for voltage determination. Moreover, LI provides a high-quality criterion (MSE) that can be transferred into $S / N$ or a relative error for inversion. The applicability could be proved on a large-scale ERT data set and checked using reciprocity analyses. In general, all three approaches provided results that are almost identical. Additionally, The lock-in approach provides an error measure, giving an idea about the estimating accuracy of the individual data and resembles the estimates from reciprocity. It needs to be checked whether this measure can be used for data inversion, either individually or in a statistic sense.

There is space for future development of both the dataloggers and the processing schemes. One could analyse the decay of the voltage curves to retrieve induced polarization properties. However, this range is often dominated by overshoots; hence IP analysis would require exact knowledge of the source signals. Beyond direct-current (frequencies of $1 \mathrm{~Hz}$ or below) one could use the instrument for controlledsource electromagnetic (CSEM) surveys that use frequencies from several $\mathrm{Hz}$ to several $\mathrm{kHz}$. However, the limited sampling rate of $1 \mathrm{kHz}$ restricts the frequency range and would require a new logging concept. The lock-in approach (and similarly stacking) is expected to work until a sufficient amount of samples are required. Therefore we expect a better performance of FFT for higher frequency; however this is very sensitive to the $1 / f$ noise characteristics and requires discrimination from the higher harmonics of power lines.

Data availability. Codes and data for this publication are available at https://doi.org/10.5281/zenodo.1167654 (Oppermann and Günther, 2018).

Author contributions. FO developed the datalogger including the control software, worked on the processing methods and prepared the manuscript with contributions from the co-author. TG analysed the field data and put the manuscript into the context of ERT.

Competing interests. The authors declare that they have no conflict of interest.

Acknowledgements. We like to thank Raphael Rochlitz, Robert Meyer, Dieter Epping and Vitali Kipke for help in producing the field data as part of the DESMEX project funded by the German Ministry of Education and Research (BMBF) under grant number 033R139D.
Edited by: Jean Dumoulin

Reviewed by: two anonymous referees

\section{References}

Agricola, T.: Großskalige 3D-Geoelektrik im Hohen Vogelsberg und auf dem Seeboden der Kinzigtalsperre (Large-scale 3D geoelectrics in the High Vogelsberg and on the lake bottom of the Kinzig dam), Diss. Johann Wolfgang Goethe-Universität Frankfurt am Main, urn:nbn:de:hebis:30:3-443077, 2016.

Albertini, A. and Kleemann, W.: Analogue and digital Lock-In techniques for very-low-frequency impedance spectroscopy, Meas. Sci. Technol., 8, 666-672, 1997.

Alfano, L.: A modified geoelectrical procedure using polar-dipole arrays - an example of application to deep exploration, Geophys. Prospect. 22, 510-525, 1974.

Alfano, L.: Dipole-dipole deep geoelectrical soundings over geological structures, Geophys. Prospect. 28, 283-296, 1980.

Andersson, M., Persson, L., Svensson, T., and Svanberg, S.: Flexible Lock-In detection system based on synchronized computer plug-in boards applied in sensitive gas spectroscopy, Rev. Sci. Instrum., 78, https://doi.org/10.1063/1.2813346, 2007.

Baker, E. B.: RF Phase-Sensitive Detector for Nuclear Magnetic Resonance Signals, Rev. Sci. Instrum., 25, 390-391, 1954.

Balasco, M., Galli, P., Giocoli, A., Gueguen, E., Lapenna, V., Perrone, A., Piscitelli, S., Rizzo, E., Romano, G., Siniscalchi, A., and Votta, M.: Deep geophysical electromagnetic section across the middle Aterno Valley (central Italy): preliminary results after the April 6, 2009 L'Aquila earthquake, B. Geofis. Teor. Appl., 52, 443-455, https://doi.org/10.4430/bgta0028, 2011.

Bengtsson, L. E.: A microcontroller-based Lock-In amplifier for sub-milliohm resistance measurements, Rev. Sci. Instrum., 83, https://doi.org/10.1063/1.4731683, 2012.

Bergmann, P., Schmidt-Hattenberger, C., Kiessling, D., Rücker, C., Labitzke, T., Henninges, J., Baumann, G., and Schütt, H.: Surface-downhole electrical resistivity tomography applied to monitoring of $\mathrm{CO}_{2}$ storage at Ketzin, Germany, Geophysics, 77, B253-B267, 2012.

Blair, D. P. and Sydenham, P. H.: Phase sensitive detection as a means to recover signals buried in noise, J. Phys. E Sci. Instrum., 8, 621-627, 1975.

Brunner, I., Friedel, S., Jacobs, F., and Danckwardt, E.: Investigation of a Tertiary maar structure using three-dimensional resistivity imaging, Geophys. J. Int., 136, 771-780, 1999.

Caracappa, A. and Thorn, C.: Target Polarization Measurements with a Crossed-Coil NMR Polarimeter, in: AIP conference proceedings, Institute of Physics (IOP) Publishing Ltd., 867-871, 2003.

Colella, A., Lapenna, V., and Rizzo, E.: High-resolution imaging of the High Agri Valley basin (Southern Italy) with Electrical Resistivity Tomography, Tectonophysics, 386, 29-40, 2004.

De Marcellis, A., Ferri, G., D’Amico, A., Natale, C. D., and Martinelli, E.: A fully-analog Lock-In amplifier with automatic phase alignment for accurate measurements of ppb gas concentrations, IEEE Sens. J., 12, 1377-1383, 2012.

Dereppe, J. M.: Phase Sensitive Detector, Rev. Sci. Instrum., 32, 979-979, 1961. 
Ferri, G., De Laurentiis, P., D’Amico, A., and Di Natale, C.: A lowvoltage integrated CMOS analog Lock-In amplifier prototype for LAPS applications, Sensor. Actuator., 92, 263-272, 2001.

Flechsig, C., Fabig, T., Rücker, C. and Schütze, C.: Geoelectrical Investigations in the Cheb Basin/W-Bohemia: An Approach to Evaluate the Near-Surface conductivity structure, Stud. Geophys. Geod., 54 , 417-437, 2010.

Friedel, S.: Über die Abbildungseigenschaften der geoelektrischen Impedanztomographie unter Berücksichtigung von endlicher Anzahl und unendlicher Genauigkeit der Meßdaten, ("Imaging properties of geoelectric impedance tomography taking into account a finite number of limited accuracy measurements"), Shaker Verlag, Aachen, ISBN 3-8265-7587-3, 2000 (in German).

Gabal, M., Medrano, N., Calvo, B., Martínez, P. A., Celma, S., and Valero, M. R.: A complete low voltage analog Lock-In amplifier to recover sensor signals buried in noise for embedded applications, Procedia Engineer., 5, 74-77, 2010.

Golden, S., Roßberg, R., and Junge, A.: The data logger Geolore and its application in lake bottom magnetotellurics, in: 17th workshop on electromagnetic induction in the earth, Hyderabad, India, 2004.

Günther, T., Musmann, P., Schaumann, G., and Grinat, M.: Imaging of a fault zone by a large-scale dc resistivity experiment and seismic structural information, Ext. abstr., 17th EAGE Near Surface, Leicester, UK, 2011.

Günther, T., Ronczka, M., and Voß, T.: Saltwater Monitoring Using Long-Electrode ERT, in: Geological Storage of $\mathrm{CO}_{2}$ - Long Term Security Aspects, edited by: Liebscher, A. and Münch, U., Springer Int. Publ., 167-182, https://doi.org/10.1007/978-3-31913930-2_8, 2015.

Holzman, J. F., Scollo, R., Lohe, H. J., Robin, F., Jäckel, H., and Erni, D.: Phase-sensitive Lock-In detection of semiconductor waveguide intensity profiles, Rev. Sci. Instrum., 76, https://doi.org/10.1063/1.2126810, 2005.

Johnson, S. L., Thomas, M. V., and Kros, C. J.: Membrane capacitance measurement using patch clamp with integrated selfbalancing Lock-In amplifier, Pflü. Arch., 443, 653-663, 2002.

Masciotti, J. M., Lasker, J. M., and Hielscher, A. H.: Digital LockIn detection for discriminating multiple modulation frequencies with high accuracy and computational efficiency, IEEE T. Instrum. Meas., 57, 182-189, 2008.

Meade, M. L.: Advances in Lock-In amplifiers, J. Phys. E Sci. Instrum., 15, 395-403, 1982.

Meade, M. L.: Lock-In amplifiers: principles and applications (No. 1), Inst. of Engineering and Technology, 1983.

Murányi, F., Simon, F., Fülöp, F., and Jánossy, A.: A longitudinally detected high-field ESR spectrometer for the measurement of spin-lattice relaxation times, J. Magn. Reson., 167, 221-227, 2004.

Naess, O. E. and Bruland, L.: Stacking methods other than simple summation, Developments in Geophysical Exploration Methods, 6, 189-223, 1985.

Oppermann, F. and Günther, T.: Codes and data for Software lockin processing, Zenodo, https://doi.org/10.5281/zenodo.1167654, 2018.

Pribnow, D. F., Schütze, C., Hurter, S. J., Flechsig, C. and Sass, J. H.: Fluid flow in the resurgent dome of Long Valley Caldera: implications from thermal data and deep electrical sounding, J. Volcanol. Geoth. Res., 127, 329-345, 2003.
Proksch, R.: Multifrequency, repulsive-mode amplitudemodulated atomic force microscopy, Appl. Phys. Lett., 89, https://doi.org/10.1063/1.2345593, 2006.

Rizzo, E., Colella, A., Lapenna, V., and Piscitelli, S.: Highresolution images of the fault controlled High Agri Valley basin (Southern Italy) with deep and shallow Electrical Resistivity Tomographies, Phys. Chem. Earth, 29, 321-327, 2004.

Ronczka, M.: Saltwater detection and monitoring using metal cased boreholes as long electrodes, PhD thesis, TU Berlin, https://doi.org/10.14279/depositonce-5131, 2015.

Roßberg, R.: Geolore: Migration from an experiment to a versatile instrument, Proceedings of the 22nd Colloquium Electromagnetic Depth Research, Décin, CR, available at: http://bib. gfz-potsdam.de/emtf/2007/ (last access: 2 February 2018), 2007.

Saam, B. T. and Conradi, M. S.: Low frequency NMR polarimeter for hyperpolarized gases, J. Magn. Reson., 134, 67-71, 1998.

Santilano, A., Godio, A., Manzella, A., Menghini, A., Rizzo, E., and Romano, G.: Electromagnetic and DC methods for geothermal exploration in Italy, state-of-the-art, case studies and future developments, First Break, 33, 81-86, 2015.

Scofield, J. H.: Frequency-domain description of a Lock-In amplifier, Am. J. Phys., 62, 129-132, 1994.

Schünemann, J., Günther, T., and Junge, A.: 3-dimensional subsurface investigation by means of large-scale tensor-type dc resistivity measurements, Ext. abstract, 4th International Symposium on Three-Dimensional Electromagnetics, Freiberg, 2007.

Schütze, C. and Flechsig, C.: Structural investigations of an active hydrothermal system beneath the Long Valley caldera, California, using DC-resistivity imaging methods, Z. Geol. Wissenschaft, 30, 119-129, 2002.

Sonnaillon, M. O. and Bonetto, F. J.: A low-cost, high-performance, digital signal processor-based Lock-In amplifier capable of measuring multiple frequency sweeps simultaneously, Rev. Sci. Instrum., 76, https://doi.org/10.1063/1.1854196, 2005.

Storz, H., Storz, W., and Jacobs, F.: Electrical resistivity tomography to investigate geological structures of the earth's upper crust, Geophys. Prospect., 48, 455-471, 2000.

Surkov, V. V. and Hayakawa, M.: ULF electromagnetic noise due to random variations of background atmospheric current and conductivity, J. Geophys. Res., 112, D11116, https://doi.org/10.1029/2006JD007788, 2007.

Tamburriello, G., Balasco, M., Rizzo, E., Harabaglia, P., Lapenna, V., and Siniscalchi, A.: Deep electrical resistivity tomography and geothermal analysis of Bradano foredeep deposits in Venosa area (Southern Italy): first results, Ann. Geophys., 51, 203-212, 2008.

Udphuay, S., Günther, T., Everett, M. E., Warden, R. R., and Briaud, J.-L.: Three-dimensional resistivity tomography in extreme coastal terrain amidst dense cultural signals: application to cliff stability assessment at the historic D-Day site, Geophys. J. Int., 185, 201-220, 2011.

Vistnes, A. I., Wormald, D. I., Isachsen, S., and Schmalbein, D.: An efficient digital phase-sensitive detector for use in electron-spinresonance spectroscopy, Rev. Sci. Instrum., 55, 527-532, 1984.

Wilson, J. W., Park, J. K., Warren, W. S., and Fischer, M. C.: Flexible digital signal processing architecture for narrowband and spread-spectrum Lock-In detection in multiphoton microscopy and time-resolved spectroscopy, Rev. Sci. Instrum., 86, https://doi.org/10.1063/1.4916261, 2015. 